\title{
Rolling tachyon in brane world cosmology from superstring field theory
}

\author{
Gary Shiu \\ Department of Physics and Astronomy, University of Pennsylvania, Philadelphia, Pennsylvania 19104 \\ S.-H. Henry Tye \\ Laboratory for Elementary Particle Physics, Cornell University, Ithaca, New York 14853
}

Ira Wasserman

Center for Radiophysics and Space Research, Cornell University, Ithaca, New York 14853

(Received 8 October 2002; published 29 April 2003)

\begin{abstract}
The pressureless tachyonic matter recently found in superstring field theory has an overabundance problem in cosmology. We argue that this problem can be solved in the brane inflationary scenario if almost all of the tachyon energy is drained (via coupling to the inflaton and matter fields) to heating the Universe, while the rest of the tachyon energy goes to a network of cosmic strings (lower-dimensional BPS D-branes) produced during the tachyon rolling at the end of inflation.

DOI: 10.1103/PhysRevD.67.083517

PACS number(s): 98.80.Cq, 11.25.Sq, 11.27.+d
\end{abstract}

In superstring theory, starting with a non-Bogomol'nyiPrasad-Sommerfield (BPS) brane or a brane-antibrane pair, a tachyon is always present. This tachyon rolls down its potential and tachyon matter appears as energy density without pressure, as pointed out by Sen [1]. In the cosmological context [2], it is easy to estimate that the pressureless tachyonic matter density is generically many orders of magnitude too big to be compatible with present day cosmological observations [3]. This implies either that tachyon rolling does not happen (i.e., is irrelevant) in the early Universe after inflation, or, instead of pressureless tachyon matter density, the tachyon potential energy goes somewhere else. In this paper, we argue for the latter.

In superstring theory, defects, or stable solitons (i.e., lower-dimensional BPS branes), are produced during tachyon rolling, also first pointed out by Sen [4]. Cosmologically, these defects will be produced [5]. Averaged over a region large compared with the typical defect separation, this defect density becomes spatially uniform, and may be identified as a part of the tachyonic matter.

In the brane world realization of superstring theory, where the standard model particles are open string modes that live on the brane while the graviton and other closed string modes live in the bulk, the brane inflationary scenario [6], where the inflaton is simply an interbrane separation, looks very robust [7-9]. In this scenario, tachyons invariably emerge towards the end of brane inflation, when the branes approach each other and collide. (Defects produced before or during inflation are inflated away.) As the tachyon rolls down its potential, it was shown that the only defects copiously produced are those that appear as cosmic strings $[9,10]$. So we expect all of the tachyon potential energy goes to heating the Universe (via its coupling to the inflaton and other light open string modes) and producing the cosmic string network. Interactions of the cosmic strings will reduce the very high initial cosmic string network density to an acceptable level [11], provided the cosmic string tension $\mu$ satisfies $G \mu$ $<10^{-6}$, which seems to be always satisfied in brane inflation $[9,10]$. The presence of cosmic strings will still give detect- able signatures in the cosmic microwave background and the gravitational wave spectral density $[9,10]$. To summarize, the overabundance of the tachyon matter density problem can be solved by draining the tachyon potential energy to (re)heating and to the cosmic string production. Below, we discuss these two mechanisms.

Consider a general Lagrangian of the form $\left(\alpha^{\prime}=1\right)$

$$
\mathcal{L}=-V(T) F(X), \quad X \equiv g^{\mu \nu} T_{, \mu} T_{, \nu},
$$

where $T(x)$ is the tachyon mode, $T_{, \mu}=\partial_{\mu} T$, and $F(X)$ can be any function at this stage. In Ref. [1], $V(T) \propto e^{-T}$ and $F(X)=\sqrt{1+X}$.

The energy momentum of Eq. (1) is

$$
T_{\mu \nu}=V(T)\left[2 F_{, X} T_{, \mu} T_{, \nu}-g_{\mu \nu} F(X)\right]
$$

where $F_{, X}=\partial F / \partial X$. We can write this in the form of the energy-momentum tensor for a perfect fluid if we identify

$$
\begin{aligned}
& \rho_{T}=V(T)\left[F(X)-2 X F_{, X}\right] \equiv V(T) D(X) \\
& p_{T}=-V(T) F(X) .
\end{aligned}
$$

We shall mostly use the tachyon Lagrangian suggested by the boundary (or background-independent) string field theory [12], in particular, its superstring version $[13,14]$, which has been used to study tachyon matter recently [15]. The nonBPS brane model (with brane tension $\sqrt{2} \tau_{p}$ ) is

$$
\begin{aligned}
& V(T)=\sqrt{2} \tau_{p} e^{-T^{2} / 2} \\
& F(X)=\frac{\sqrt{\pi} \Gamma(1+X)}{\Gamma(X+1 / 2)}=\frac{X 4^{X} \Gamma(X)^{2}}{2 \Gamma(2 X)} .
\end{aligned}
$$

For spatially independent solutions, $p_{T}<0$ for $\dot{T}^{2}<1 / 2$ and $p_{T}>0$ for $\dot{T}^{2}>1 / 2$. As $\dot{T}^{2}=-X \rightarrow 1, F(X) \simeq-1 / 2\left(1-\dot{T}^{2}\right)$, $D(X) \simeq\left(1-\dot{T}^{2}\right)^{-2}, \quad$ so $\quad w_{T}=p_{T} / \rho_{T}=-F(X) / D(X) \simeq(1$ $\left.-\dot{T}^{2}\right) / 2 \rightarrow 0$. The effective tachyon sound speed $v_{T}$ 
$=\sqrt{-F_{, X} / D_{, X}} \rightarrow 0$ for $\dot{T}^{2} \rightarrow 1$, allowing gravitational clustering. Since $w_{T}$ and $v_{T}$ both vanish as $\dot{T}^{2} \rightarrow 1$, tachyon matter is possible cold dark matter candidate, but substantial finetuning is necessary for tachyon dark matter to be viable [3].

Next, let us consider the brane-antibrane pair, which has a complex tachyon $T=T_{1}+i T_{2}$. The tachyon action is [14]

$$
\mathcal{S}_{T}=-2 \tau_{p} \int d x^{p+1} e^{-\left(T_{1}^{2}+T_{2}^{2}\right) / 2} F\left(X_{1}\right) F\left(X_{2}\right)
$$

where $X_{I} \equiv g^{\mu \nu} \partial_{\mu} T_{I} \partial_{\nu} T_{I}$. Again, let us consider spatially independent solutions. The energy conservation requires that $\dot{\rho}_{T}=0$, where

$$
\rho_{T}=2 \tau_{p} e^{-|T|^{2} / 2}\left[F_{1} F_{2}-2 X_{1} F_{, X_{1}} F_{2}-2 X_{2} F_{1} F_{, X_{2}}\right]
$$

where $F_{I}=F\left(X_{I}\right)$ and $F_{, X I}=F_{, X}\left(X_{I}\right)$. First, consider a simple ansatz of linear tachyon profile

$$
T_{1}(t)=t-\epsilon(t)
$$

and $T_{2}=0$. One finds that

$$
\dot{\epsilon}(t) \simeq e^{-t^{2} / 4} \rightarrow 0
$$

and $1+X_{1} \rightarrow 0$, so $w_{T}=0$ (i.e., zero pressure). Since $X_{2}=0$ and $F\left(X_{2}\right)=1$, this essentially reduces to the single $T$ case. In this solution, pressure approaches zero from the positive side [15].

Suppose we consider a more symmetric ansatz: $T_{1}(t)=t$ $-\epsilon_{1}(t)$, and $T_{2}(t)=t-\epsilon_{2}(t)$. An examination of the equations of motion yields $\dot{\epsilon}_{1} \propto \dot{\epsilon}_{2} \propto e^{-t^{2} / 3}$. However, when these are substituted into $\rho_{T}$, we find

$$
\rho_{T} \simeq-\frac{\tau_{p} e^{-t^{2}}}{8}\left(\frac{1}{\dot{\epsilon}_{1}^{2} \dot{\epsilon}_{2}}+\frac{1}{\dot{\epsilon}_{1} \dot{\epsilon}_{2}^{2}}\right) .
$$

A positive constant $\rho_{T}$ implies $\dot{\epsilon}_{I}<0$ (i.e., $1+X_{I}<0$ ), a solution that cannot be reached starting from small $\dot{T}_{I}$.

Let us estimate the tachyon matter density today as a fraction $\Omega_{T}$ of the total density $3 H_{0}^{2} M_{P l}^{2}$ of our Universe. We assume $\rho_{\text {, initial }} \simeq M_{s}^{4}$, where the string scale $M_{s}^{2}=1 / \alpha^{\prime}$. Using $H_{0}^{2} / M_{P l}^{2} \simeq 10^{-123}$ and $a_{\text {initial }} / a_{\text {today }} \simeq 2.7^{\circ} \mathrm{K} / M_{s}$, we find

$$
\Omega_{T}=\frac{\rho_{T, \text { initial }}}{3 H_{0}^{2} M_{P l}^{2}}\left(\frac{a_{\text {initial }}}{a_{\text {today }}}\right)^{3} \simeq 10^{28} \frac{M_{s}}{M_{P l}}
$$

where $2.7^{\circ} \mathrm{K} / M_{P l} \simeq 10^{-31}$. Since $\Omega_{T}$ must be less than 1 , and $M_{s}>1 \mathrm{TeV}$, we see that the tachyon matter density will be many orders of magnitude too big to be compatible with our Universe [3]. Fortunately, as we shall argue below, this tachyon energy can be completely drained by cosmic string production and (re)heating in a realistic early Universe.

One may also consider a stable solitonic solution of Eq. (5), with $T_{1}(x)=u_{1} x_{1}$ and $T_{2}(x)=u_{2} x_{2}$ [14]. Since both the second derivatives of $T_{I}(x)$ and the mixed derivatives $\partial_{i} T_{1} \partial_{j} T_{2}$ with $i=j$ vanish in this ansatz, the equations of motion reduce to $T_{I} D\left(X_{I}\right)=0(I=1,2)$. Since $D\left(X_{I}\right)$ only vanishes at $X_{I}=u_{I}^{2} \rightarrow \infty$ (this means infinitely thin brane), the codimension-2 brane tension $[F(X) \rightarrow \sqrt{\pi X}]$ becomes

$$
\begin{aligned}
\tau_{p-2} & =2 \tau_{p} \int d x_{1} d x_{2} e^{-|T|^{2} / 2} F\left(u_{1}^{2}\right) F\left(u_{2}^{2}\right) \\
& =2 \tau_{p} F\left(u_{1}^{2}\right) F\left(u_{2}^{2}\right) 2 \pi / u_{1} u_{2} \rightarrow \tau_{p}(2 \pi)^{2}
\end{aligned}
$$

as expected. This is a vortex solution.

A non-BPS $(p-1)$-brane may also be produced $\left(u_{1}\right.$ $\left.\rightarrow 0, u_{2} \rightarrow \infty\right)$, but it will quickly decay to BPS $\mathrm{D}(p-2)$-branes. A moving brane with constant velocity $v$ may also be constructed by generalizing $T_{1}$ to $T_{1}(x)$ $=u_{1}\left(x_{1}-v t\right)$, which gives the energy $E=\tau_{p-2} / \sqrt{1-v^{2}}$, as expected.

If we start with a brane-antibrane pair, the tachyon will roll with non-trivial spatial dependence. This will result in a collection of $\mathrm{D}(p-2)$-branes. However, if we average over a region much larger than the typical spacing between the $\mathrm{D}(p-2)$-branes, we expect to obtain a spatially uniform energy density and isotropized pressure, that is, a perfect fluid. In this sense, we may interpret the density of defects (which turn out to be cosmic strings $[9,10]$ ) as part of the tachyon matter. The Kibble mechanism would imply an initial density $\sim H^{2} M_{s}^{2} \sim M_{s}^{6} / M_{P l}^{2}$ in cosmic strings at the end of brane inflation. Intercommutation of intersecting cosmic stings and the decay of string loops (to gravitational radiation) causes the density of the cosmic string network (CSN) to approach $\Omega_{C S N} \sim G \mu \sim M_{S}^{2} / M_{P l}^{2}$ [11], which would be acceptable. However, the initial density of the network is only a fraction $\sim M_{s}^{2} / M_{P l}^{2}$ of the total density of the Universe, $\sim M_{s}^{4}$, at the end of brane inflation. This starting density must be overwhelmingly in the form of radiation in order for the cosmology to be viable. Thus, we must understand how the mass density in tachyons can convert to radiation efficiently at the end of brane inflation.

To drain the tachyon energy to heat the Universe, we need to couple the tachyon field to other fields, the most obvious one being the scalar field $\phi$ that describes the separation between the brane and the antibrane $(\phi=0$ in the above discussion). In brane inflation, $\phi$ is the inflaton. Let us take a simple generalization of the above tachyon model to include the inflaton $\phi$,

$$
\mathcal{L}(T, \phi)=-V(|T|, \phi) F\left(X_{1}\right) F\left(X_{2}\right)-\frac{1}{2} g^{\mu \nu} \phi_{, \mu} \phi, \nu .
$$

For brane inflation, the inflaton potential is known to have the form $U(\phi) \simeq U_{0}-U_{1} / \phi^{d-2}$ for large $\phi$, when there are $d$ extra dimensions transverse to the brane $[7,16]$. For a particular brane pair, $U_{i}$ are easily calculable [17]. For example, for a brane-antibrane pair, $U_{0}=2 \tau_{p} V_{p-3}$ where $V_{p-3}$ is the compactification volume of the extra $(p-3)$ dimensions of the branes and $U_{1}$ is simply twice that of the gravitational force. When the brane separation $\phi$ is comparable to the compactification size, the compactification effect becomes 
important and it flattens the potential further [7,9], allowing inflation to take place for many $e$-foldings.

When the branes are far apart, $T$ is a normal scalar field with positive mass squared $m_{T}^{2}$. As the branes move closer, $m_{T}^{2}$ decreases, and becomes negative at the bifurcation point $\phi_{v}$, where $T$ becomes tachyonic. This suggests the following potential:

$$
V(|T|, \phi)=U(\phi) \exp \left[-|T|^{2}\left(\frac{1}{2}-\lambda \phi^{2}\right)\right]
$$

where the bifurcation point $\lambda \phi_{v}^{2}=1 / 2$.

Although the potential $U(\phi)$ for small $\phi$ remains to be calculated, it is expected to be positive and finite as $\phi \rightarrow 0$. In terms of open strings, $U(\phi=0)$ is a quantum one-loop correction, so $2 \tau_{p}$ is renormalized to $2 \tau_{p} v_{0}$, where we shall take $1>v_{0}>0$. So, for $\phi=0$, we recover the tachyonic model (5). Notice that the minimum of the potential $V(|T|, \phi) \quad\left(\phi<\phi_{v}\right.$ and $\left.T \rightarrow \infty\right)$ is automatically zero, independent of the details of $U(\phi)$. As a consequence, the dynamics is insensitive to the particular form of $U(\phi)$ for $\phi$ $<\phi_{v}$, since tachyon rolling happens very fast, and $V(|T|, \phi) \rightarrow 0$ rapidly. For phenomenology, we choose a particularly simple form of $U(\phi)$, where $U(\phi)$ has a simple harmonic form for small $\phi$,

$$
U(\phi)=U_{0}\left[1-\frac{\left(1-v_{0}\right)}{\left(\phi^{2} / \phi_{0}^{2}+1\right)^{(1 / 2)(d-2)}}\right] .
$$

In summary, Eqs. (12),(13),(14) comprise the model, which may be considered as a novel version of hybrid inflation.

For fixed $\phi>\phi_{v}, T$ has positive mass $m_{T}^{2}>0$, so the minimum of the potential is at $T_{I}=0$ and classically $\partial_{\mu} T_{I}$ $=0$, so $X_{I}=0$ and $F\left(X_{I}\right)=1$. The model reduces to $\mathcal{L}(\phi, T=0)=-U(\phi)-g^{\mu \nu} \partial_{\mu} \phi \partial_{\nu} \phi / 2$. This describes the inflationary epoch for $\phi>\phi_{v}$.

Based on the above analysis, let only one of the tachyons roll, say $T=T_{1}$, while $T_{2}$ is essentially frozen, with $F\left(X_{2}\right)$ $=1$. The analysis applies just as well if we replace $F\left(X_{1}\right) F\left(X_{2}\right)$ in Eq. (12) with the $\mathrm{U}(1)$ invariant $F\left(X_{1}\right.$ $\left.+X_{2}\right)$. For a spatially uniform scalar field and tachyon in a FRW expanding Universe,

$$
\begin{aligned}
\ddot{\phi}+\left(3 H+\Gamma_{\phi}\right) \dot{\phi} & =-F(X)[V(|T|, \phi)]_{,} \\
\ddot{T}+3 H A(X) \dot{T} & =-\frac{V_{, T}}{2 V} B(X)-\frac{V_{, \phi}}{V} A(X) \dot{T} \dot{\phi},
\end{aligned}
$$

where $\quad A(X)=F_{, X} /\left(F_{, X}+2 X F_{, X X}\right) \quad$ and $\quad B(X)=(F$ $\left.-2 X F_{, X}\right) /\left(F_{, X}+2 X F_{, X X}\right) ; \Gamma_{\phi}$ is a phenomenological term that models the decay of $\phi$ to ordinary particles and radiation, whose density is $\rho_{\text {matter }}$. If the inflaton decays primarily to relativistic particles ("radiation"), then

$$
\dot{\rho}_{\text {matter }}+4 H \rho_{\text {matter }}=\Gamma_{\phi} \dot{\phi}^{2} .
$$

The total energy density in this situation is

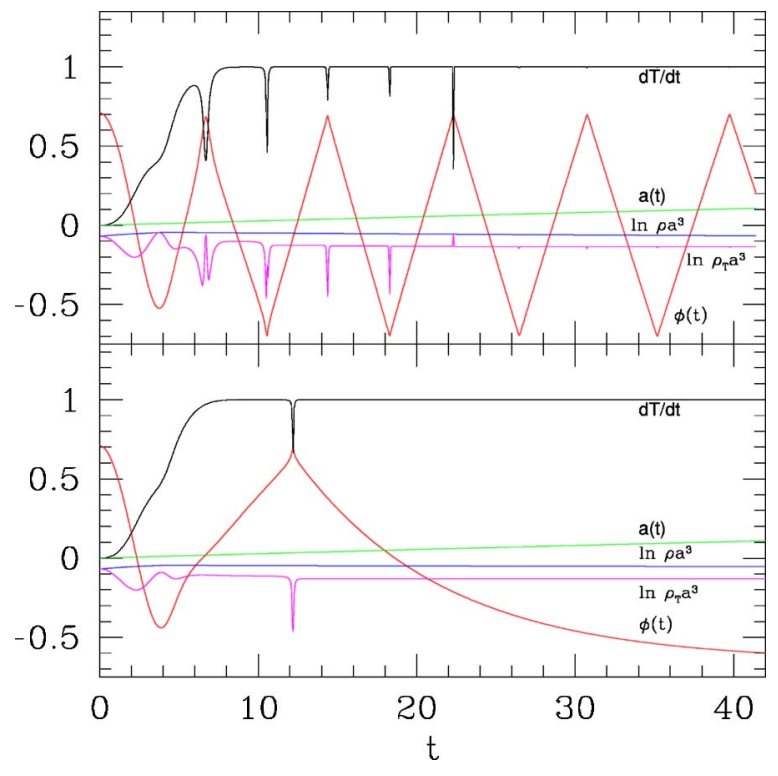

FIG. 1. Cosmological evolution of $\phi(t), \dot{T}, \ln a(t), \ln \rho a^{3}$ and $\ln \rho_{T} a^{3}$ for $\Gamma_{\phi}=0$ (top) and $\Gamma_{\phi}=0.1$ (bottom). $M_{P l}=10^{3}, v_{0}$ $=0.9, U_{0}=1$.

$$
\rho=V(|T|, \phi)\left(F(X)+2 \dot{T}^{2} F_{, X}\right)+\frac{\dot{\phi}^{2}}{2}+\rho_{\text {matter }}
$$

and $H^{2}=8 \pi G \rho / 3$. Here, we are not interested in the entire process of brane inflation, just what happens at the end, when the tachyon starts to roll. We assume that there is a prior period of slow rolling ( $\phi$ decreasing slowly), during which almost all of the inflation occurs, and density fluctuations are generated [7-9]. During the inflationary period, when $|T| \phi$ is small and (let $\lambda=1$ ) $\phi>2^{-1 / 2}$, the evolution of $T(t)$ resembles a massive field, with a mass $\sim \phi$, and we therefore expect $T(t)$ to remain small, and dominated by quantum fluctuations. When the tachyon becomes unstable, it begins with an amplitude $T \sim H_{I} / 2 \pi \sqrt{U_{0}} \sim M_{P l}^{-1}$ and $\dot{T}$ $\sim H_{I} T$, where $H_{I}=\left(8 \pi G U_{0} / 3\right)^{1 / 2}$ is the expansion rate during slow roll. We follow the evolution implied by Eqs. (15) and (16) after $\phi=2^{-1 / 2}$.

As an illustration, numerical results are shown in Fig. 1, assuming (in terms of string scale) $1 / M_{P l}=10^{-3}, U_{0}=1$, $1-v_{0}=0.1, d=4, \phi_{0}=1$, and $\Gamma_{\phi}=0$ or 0.1 . In fact, because $H_{I} \simeq 0.003$, cosmological expansion is relatively unimportant. The simulations start with $\phi(t)=1 / \sqrt{2}$ and $\dot{\phi}=0$, at $t \equiv 0$ and $a(t) \equiv 1$. The initial data can be varied, but the behavior shown in Fig. 1 is generic. At first, $\phi(t)$ decreases, and $\dot{T}$ increases. Ultimately, as $\dot{T} \rightarrow 1, T \simeq t$ grows, and the product $V(T, \phi) F(X)$ decreases rapidly for $\phi^{2}<1 / 2$. In the nondissipative case, $\Gamma_{\phi}=0, \dot{\phi} a^{3} \rightarrow$ const, and since $a(t)$ is only changing slowly, $\phi$ increases or decreases linearly with time. However, since the total energy is conserved, and $V(T, \phi)$ would grow exponentially large for $\phi^{2}>1 / 2$, the growth of $|\phi|$ cannot continue indefinitely, so $\phi(t)$ bounces near $\phi^{2}=1 / 2$. Associated with these bounces are short-lived dips in $\dot{T}$, which are evident but not completely resolved in 
Fig. 1. Eventually, cosmological expansion alone would cause $\dot{\phi}$ to decay, and $\phi$ would settle to some constant, nonzero value. Dissipation accelerates the decay of the nonlinear oscillations. The bottom panel in Fig. 1 shows what happens for $\Gamma_{\phi}=0.1$, which may be unrealistically large, but illustrates how dissipation suppresses the oscillations, and causes $\phi$ to settle to a nonzero value asymptotically. For either $\Gamma_{\phi}$, $\rho_{T} a^{3} \rightarrow$ const $\sim 1$ eventually, where $\rho_{T} \equiv V(T, \phi) D(X)$. For $\Gamma_{\phi}=0.1, \rho_{T} / \rho \geqslant 0.9$ at the end of the numerical integration; the Universe does not become radiation dominated.

Radiation domination can be achieved if the tachyon couples directly to matter. A possible mechanism might be tachyon preheating [18]. To explore a different possibility phenomenologically, suppose we add a dissipative term $-\Gamma_{T} \dot{T}$ to the second of Eqs. (15), and assume that the energy loss due to this term goes directly to matter. Then the rate of increase of the matter density due to this form of coupling is $2 \Gamma_{T} X D_{X} V(T, \phi)$. Assuming that the inflaton, $\phi$, also couples to matter as in the above model, we find that eventually $\phi \rightarrow \phi_{\infty}$, with $\left|\phi_{\infty}\right|<1 / \sqrt{2}$, while $\dot{\phi} \rightarrow 0$. The tachyon rolls dissipatively toward its minimum at late times, and balancing the drag $-\Gamma_{T} \dot{T}$ with the acceleration down the tachyon potential, $-V_{, T} B(X) / 2 \mathrm{~V}$, implies that

$$
1-\dot{T} \simeq \frac{2 \Gamma_{T}}{t\left(1-2 \phi_{\infty}^{2}\right)} .
$$

Thus, $1+X \simeq 2(1-\dot{T}) \rightarrow 0$ asymptotically, but only as a power of time, whereas $V(T, \phi) \simeq U\left(\phi_{\infty}\right) \exp \left[-t^{2}(1 / 2\right.$ $\left.-\phi_{\infty}^{2}\right)$ ] drops exponentially; the rate of decrease of $1+X$ is far slower than in Eqs. (7) and (8). After a time of order the maximum of $\Gamma_{T}^{-1}$ and $\Gamma_{\phi}^{-1}$, the Universe becomes radiation dominated, and the tachyon matter density is exponentially small. Depending on $\Gamma_{T}$ and $\Gamma_{\phi}$, there could be an early phase of tachyon domination, but it would not last long enough to have any important, lasting effects. Although tachyon decay to matter never ceases in this model, the radiation production rate becomes exponentially small at late times, and the additional heating long after the end of inflation is inconsequential.

It is possible that $\Gamma_{T}$ is a function of $T$, not constant. Eq. (18) already gives some idea of what might happen. If $\Gamma_{T}$ decreases with increasing $T$, then we expect that eventually dissipation becomes relatively unimportant, and the Universe may be left with a residual tachyon matter density. On the other hand if $\Gamma_{T}$ increases with increasing $T$, then we might expect $\dot{T}$ to fall toward zero eventually, with $T$ asymptoting to some constant value or else growing very slowly.

Let us consider first what happens when $\Gamma_{T}$ is a decreasing function of $T$. Suppose, as is plausible, that $\Gamma_{\phi}$ is characterized by the string scale, so that $\phi$ tends toward a timeindependent value as branes approach one another relatively quickly, perhaps even faster than expansion. For $H$ $\sim M_{s}^{2} / M_{P l}$, and $\Gamma_{\phi}=\beta M_{s}$, where $\beta$ is dimensionless, $\Gamma_{\phi} / H \sim \beta M_{P l} / M_{s}$, which is greater than one as long as $\beta$ $\geq M_{s} / M_{P l}$. As $\dot{\phi} \rightarrow 0$ and $\dot{T} \rightarrow 1$, the evolution equation for $\rho_{T}$ simplifies to

$$
\dot{\rho}_{T}+3 H \rho_{T} \simeq-\frac{V(T) \Gamma_{T}(T)}{2(1-\dot{T})^{3}} \simeq-\frac{4 \Gamma_{T}(T) \rho_{T}^{3 / 2}}{\sqrt{V(T)}},
$$

where we used $\rho_{T} \simeq V(T) / 4(1-\dot{T})^{2}$ to obtain the last form of the RHS. Equation (19) has the solution

$$
\rho_{T} a^{3} \simeq\left[u_{\phi}+2 \int_{t_{\phi}}^{t} \frac{d t^{\prime} \Gamma_{T}\left(t^{\prime}\right)}{\left[a\left(t^{\prime}\right)\right]^{3 / 2} \sqrt{V\left(t^{\prime}\right)}}\right]^{-2},
$$

where $u_{\phi}$ is the value of $\left(\rho_{T} a^{3}\right)^{-1 / 2}$ at roughly the time $t_{\phi}$ when $\phi$ freezes; assuming a density $\sim M_{s}^{4}$ and scale factor $\sim T_{0} / M_{s}$, where $T_{0}$ is the present temperature of the Universe, $u_{\phi} \sim\left(M_{s} / T_{0}\right)^{3 / 2} \sim 10^{47.6}\left(M_{s} / M_{P l}\right)^{3 / 2}$. In obtaining Eq. (20), we have approximated $T(t) \simeq t$ in the integrand. Equation (18) can be recovered from Eq. (20) by evaluating the integral asymptotically under the approximation that $\Gamma_{T}$ is independent of $T$, and expansion is slow compared to the string time scale. For constant $\Gamma_{T}$, the tachyon matter density falls rapidly with time, roughly like $\exp \left[-t^{2}(1 / 2\right.$ $\left.-\phi_{\infty}^{2}\right)$ ], and becomes utterly negligible. As long as $\Gamma_{T}$ does not fall faster than $\sqrt{V(T)}$, then the integral in Eq. (20) grows very rapidly. In general, Eq. (20) implies that for an acceptable $\rho_{T}$ today

$$
\begin{aligned}
\int_{t_{\phi}}^{t_{0}} \frac{d t^{\prime} \Gamma_{T}\left(t^{\prime}\right)}{\left[a\left(t^{\prime}\right)\right]^{3 / 2} \sqrt{V\left(t^{\prime}\right)}} & \geq 10^{14} \sqrt{\frac{M_{s}}{\Omega_{T} M_{P L}}} u_{\phi} \\
& \sim 10^{61.6} \Omega_{T}^{-1 / 2}\left(\frac{M_{s}}{M_{P l}}\right)^{2},
\end{aligned}
$$

if $\Gamma_{T}$ is a decreasing function of $T ; t_{0} \gg t_{\phi}$ is the present age of the Universe. Although it is possible for tachyon matter to become dominant in models with decreasing $\Gamma_{T}$, extreme fine-tuning is required in order for tachyons to be the cold dark matter. Since $1 / \sqrt{V(t)}$ increases so rapidly-like a Gaussian in $t$-decay of the tachyon field would have to shut off completely after only $\sim 10 M_{s}^{-1}$ for Eq. (21) to allow substantial $\rho_{T}$ today. Otherwise, the value of $\rho_{T}$ today ought to be utterly negligible. Generically, we expect that $\Gamma_{T}$ cannot shut off effectively and quickly enough to leave a substantial tachyon matter density today.

It is also conceivable that $\Gamma_{T}$ depends on both $T$ and $\dot{T}$. As $\dot{T} \rightarrow 1$, suppose that $\Gamma_{T}=(1-\dot{T})^{p} \gamma_{T}(T)$. Then instead of Eq. (20) we would find

$$
\rho_{T} a^{3} \simeq\left[u_{\phi}^{1-p}+2^{1-p} \int_{t_{\phi}}^{t} \frac{d t^{\prime}(1-p) \gamma_{T}\left(t^{\prime}\right)}{\left[a^{3}\left(t^{\prime}\right) V\left(t^{\prime}\right)\right]^{(1-p) / 2}}\right]^{-2 /(1-p)} .
$$

For $p<1$, Eq. (22) is similar to Eq. (21), and implies a leftover tachyon matter density at late times which may or may not be important depending on the details of the damping rate. For $p>1$, the integrand in Eq. (22) is proportional to $[V(t)]^{(p-1) / 2}$, which falls like a Gaussian, so we expect relatively little decrease in $\rho_{T} a^{3}$, for $\gamma_{T} \leqslant 1$. The special value $p=1$ corresponds to a dissipation rate proportional to $\rho_{T}$, and in that case we find 


$$
\rho_{T} a^{3} \simeq\left(\rho_{T} a^{3}\right)_{\phi} \exp \left[-2 \int_{t_{\phi}}^{t} d t^{\prime} \gamma_{T}\left(t^{\prime}\right)\right]
$$

The final value of $\rho_{T} a^{3}$ depends sensitively on how quickly $\gamma_{T}(T)$ decreases with increasing $T$ for $p=1$.

Next, let us briefly consider the opposite case, where $\Gamma_{T}$ increases with $T$ faster than $T$. In that case, $\dot{T}$ eventually drops toward zero, and $T(t)$ changes only slowly. If the dissipative drag approximately balances acceleration down the tachyon potential then once $\phi$ has frozen

$$
\Gamma_{T} \dot{T} \simeq \frac{T\left(1-2 \phi_{\infty}^{2}\right)}{2 \ln 4} .
$$

Small $\dot{T}$ requires small $T / \Gamma_{T}$. For this regime to extend to small $\rho_{T} \simeq V(T)$, we would have to require that $\Gamma_{T} / T$ is large even for moderately large values of $T$. But this would also imply that $\Gamma_{T}$ becomes large in units of the string scale, which would not be expected to occur in perturbation theory; in a nonperturbative calculation, back reaction ought to prevent the growth of $\Gamma_{T}$ to large values. Thus, if $\Gamma_{T}$ increases at all, it must only do so for a short time, lasting only $\sim M_{S}^{-1}$. Thereafter, $\Gamma_{T}$ could level off to a constant value or decrease, and the physics reverts to Eq. (20) [or Eq. (18)].

Possible products of tachyon decay could include ordinary elementary particles, gravitational radiation and massive defects. Of these, ordinary elementary particles would be the most benign, since they would simply thermalize to reheat the Universe. Since most of the decrease in tachyon energy density must occur within $\sim 10 M_{s}^{-1}$ of brane collision, the wavelengths of any gravitons produced are expected to be small, at most of order $\sim \zeta M_{P l} / M_{s} T_{0}$ $\sim 0.1 \zeta M_{P l} / M_{s} \mathrm{~cm}$, where $\zeta \sim 10$ or so. This estimate presumes that the Universe becomes radiation dominatedmaybe by gravitational radiation predominantly-at a density $\sim\left(M_{s} / \zeta\right)^{4}$, and that gravitational radiation produced at this epoch has wavelengths smaller than the horizon scale $\sim \zeta^{2} M_{P l} / M_{s}^{2}$, which redshifts by a factor of $\sim M_{s} / \zeta T_{0}$ to the present day. The most stringent limit on the density of short wavelength gravitational radiation comes from the requirement that the energy density in gravitons is sufficiently small at the time of nucleosynthesis. Suppose that the end result of reheating is a fraction $f_{g}$ in gravitons, with the rest in ordinary elementary particles (perhaps produced only by the decay of the inflaton). If the effective number of spin degrees of freedom of relativistic particles in equilibrium after reheating is $S_{r h}$, and $S_{n u c}$ is the analogous value at nucleosynthesis, then we expect that the fraction of the energy density in gravitons at nucleosynthesis is $\simeq f_{g}\left(S_{n u c} / S_{r h}\right)^{4 / 3}$. ( $S_{\text {nuc }}=43 / 8$ before neutrinos go out of equilibrium at $T$ $\simeq 1 \mathrm{MeV}, S_{\text {nuc }}=11 / 4$ between $1 \mathrm{MeV}$ and $e^{ \pm}$annihilation at $\sim m_{e}$, and $S_{n u c}=1$ after $e^{ \pm}$annihilation.) Assuming that gravitons can only comprise at most $\epsilon_{n u c}$ of the total energy density of the Universe at the nucleosynthesis epoch implies that $f_{g}\left(S_{n u c} / S_{r h}\right)^{4 / 3} \leq \epsilon_{n u c}$; for $\epsilon_{n u c} \sim 0.01-0.1$, this limit can be satisfied as long as $S_{r h} / S_{n u c} \gtrsim 10-100$, which is not implausibly large. Massive defects that decay to elementary particles or gravitons could also be acceptable, provided that they decay early enough.

This model does not account for any small scale inhomogeneities in $T(\mathbf{x}, t)$, and so does not describe formation of cosmic strings. The decay of the tachyon energy density is not quite as efficient as outlined above because cosmic strings form via the Kibble mechanism. However, the density of cosmic strings forming in this way is expected to be small, and, at least within the context of the $\Gamma_{T} \neq 0$ model described above, could be far smaller than the density in radiation at the end of brane inflation, and small enough not to be ruled out by currently available cosmological observations.

To summarize, we have shown that the tachyon matter density problem can be solved via strong enough dissipation and reheating. Generically, such mechanisms, if active, ought to result in a negligible tachyon matter density today; extreme fine-tuning is needed for tachyon matter to be the cold dark matter. Equation (21) gives a numerical constraint that must be satisfied by such a mechanism. Whether or not there are realistic physical scenarios to achieve the required tachyon decay remains to be seen.

We thank J. Cline, N. Jones, L. Kofman, A. Linde, A. Naqvi, A. Sen and S. Shatashvili for discussions. This research is partially supported by the DOE grant EY-76-023071 (G.S.), UPenn SAS Dean's fund (G.S.), NSF (S.-H.H.T.), and NASA (I.W.).
[1] A. Sen, J. High Energy Phys. 04, 048 (2002); 07, 065 (2002); Mod. Phys. Lett. A 17, 1797 (2002).

[2] G.W. Gibbons, Phys. Lett. B 537, 1 (2002); A. Frolov, L. Kofman, and A. Starobinsky, ibid. 545, 8 (2002); L. Kofman and A. Linde, J. High Energy Phys. 07, 004 (2002).

[3] G. Shiu and I. Wasserman, Phys. Lett. B 541, 6 (2002).

[4] A. Sen, J. High Energy Phys. 08, 012 (1998); E. Witten, ibid. 12, 019 (1998); P. Horava, Adv. Theor. Math. Phys. 2, 1373 (1999).

[5] G.W. Gibbons, K. Hori, and P. Yi, Nucl. Phys. B596, 136 (2001); M. Majumdar and A.-C. Davis, J. High Energy Phys. 03, 056 (2002); K. Hashimoto, ibid. 07, 035 (2002).

[6] G.R. Dvali and S.-H.H. Tye, Phys. Lett. B 450, 72 (1999).

[7] C.P. Burgess, M. Majumdar, D. Nolte, F. Quevedo, G. Rajesh, and R.J. Zhang, J. High Energy Phys. 07, 047 (2001).

[8] J. Garcia-Bellido, R. Rabadan, and F. Zamora, J. High Energy Phys. 01, 036 (2002); C. Herdeiro, S. Hirano, and R. Kallosh, ibid. 12, 027 (2001); B.S. Kyae and Q. Shafi, Phys. Lett. B 526, 379 (2002).

[9] N. Jones, H. Stoica, and S.-H.H. Tye, J. High Energy Phys. 07, 051 (2002).

[10] S. Sarangi and S.-H.H. Tye, Phys. Lett. B 536, 185 (2002).

[11] A. Albrecht and N. Turok, Phys. Rev. Lett. 54, 1868 (1985); D.P. Bennett and F.R. Bouchet, ibid. 60, 257 (1988); B. Allen and E.P.S. Shellard, ibid. 64, 119 (1990).

[12] E. Witten, Phys. Rev. D 46, 5467 (1992); 47, 3405 (1993); S. Shatashvili, Phys. Lett. B 311, 83 (1993).

[13] D. Kutasov, M. Marino, and G.W. Moore, hep-th/0010108. 
[14] P. Kraus and F. Larsen, Phys. Rev. D 63, 106004 (2001); T. Takayanagi, S. Terashima, and T. Uesugi, J. High Energy Phys. 03, 019 (2001).

[15] S. Sugimoto and S. Terashima, J. High Energy Phys. 07, 025 (2002); J.A. Minahan, ibid. 07, 030 (2002).

[16] G. Dvali, Q. Shafi, and S. Solganik, hep-th/0105203.
[17] See, e.g., J. Polchinski, String Theory (Cambridge University Press, Cambridge, England, 1998).

[18] G. Felder, J. Garcia-Bellido, P.B. Greene, L. Kofman, A. Linde, and I. Tkachev, Phys. Rev. Lett. 87, 011601 (2001); G. Felder, L. Kofman, and A. Linde, Phys. Rev. D 64, 123517 (2001). 